\title{
INFLAMMATORY BOWEL DISEASE
}

\section{Nodular regenerative hyperplasia in patients with inflammatory bowel disease treated with azathioprine}

\author{
G Vernier-Massouille, J Cosnes, M Lemann, P Marteau, W Reinisch, D Laharie, G Cadiot, Y Bouhnik, \\ M De Vos, A Boureille, B Duclos, P Seksik, J-Y Mary, J-F Colombel
}

See end of article for authors' affiliations

Correspondence to:

J F Colombel, Department of Hepato-Gastroenterology, Hôpital Claude Huriez, CH et U de Lille, France; jfcolombel@chru-lille.fr

Revised 3 May 2007

Accepted 7 May 2007

Published online first

15 May 2007

\begin{abstract}
Aim: To assess the characteristics and clinical course of nodular regenerative hyperplasia (NRH) in patients with inflammatory bowel disease treated with azathioprine, so as to estimate the frequency of this complication and search for risk factors.

Methods: Cases were identified through a systematic survey of patients followed at 11 centres. At one centre, the cumulative risk of $\mathrm{NRH}$ was estimated and a case-control study was undertaken to identify risk factors. Results: 37 cases of NRH (30 male, 7 female) were identified between 1994 and 2005. The median dose of azathioprine was $2 \mathrm{mg} / \mathrm{kg} / \mathrm{d}$ (range 1.5 to 3.0). The median time between the start of azathioprine and the diagnosis of NRH was 48 months (range 6 to 187). After a median follow up period of 16 months (range 1 to 138), 14 patients developed complications of portal hypertension. Using multivariate analysis, male sex and stricturing behaviour were the two risk factors associated with $\mathrm{NRH}$ in patients treated with azathioprine. The cumulative risk calculated from the database (one centre) was $0.5 \%$ at 5 years (95\% confidence interval, 0.11 to 0.89 ) and $1.25 \%$ at 10 years $(0.29$ to 2.21$)$.

Conclusions: NRH is a rare but potentially severe complication of azathioprine in patients with inflammatory bowel disease. Clinicians should be aware of this complication, and should monitor liver function tests and platelet counts closely in their patients.
\end{abstract}

$\mathrm{N}$ odular regenerative hyperplasia (NRH) is a rare hepatic lesion defined by diffuse nodulation of the hepatic parenchyma, without annular fibrosis, corresponding to alternating atrophic and hyperplastic areas. ${ }^{1-3}$ Its aetiology and natural history are poorly understood; indeed, only about 200 cases have been reported. Symptoms, when present, are mainly associated with the complication of portal hypertension. ${ }^{245}$ Among drugs likely to cause NRH are the purine analogues azathioprine, 6-mercaptopurine (6-MP), and 6-thioguanine (6TG). Drug surveillance data have yielded several cases of NRH following renal, hepatic, or bone marrow transplantation. ${ }^{26-14}$ Nevertheless, the role of azathioprine remains uncertain because there are other causes of liver damage in transplant patients.

Hepatotoxicity from azathioprine or 6-MP usually presents as an increase in transaminase levels. This phenomenon has been linked to the ascribed toxicity of high levels of 6-methyl mercaptopurine metabolites. Toxicity is usually not severe, and a dose reduction is effective in most patients. ${ }^{15}$ In contrast, the onset of NRH in patients treated with azathioprine/6-MP for inflammatory bowel disease would appear to be a rare event, as only eight cases have been reported up to now. ${ }^{9}{ }^{16-19}$ This small number of NRH cases is surprising given the larger number of cases reported in renal transplant patients treated with azathioprine. Concern about this complication in inflammatory bowel disease has been rekindled following recent experience with 6-TG in the treatment of Crohn's disease. 6-TG is an attractive drug for the treatment of inflammatory bowel disease, as its use prevents the formation of intermediate metabolites of azathioprine and 6-MP which cause allergic complications, and allows high concentrations of 6-thioguanine nucleotides (6-TGN) - which are responsible for the therapeutic efficacy-to be obtained. Promising initial results of 6-TG in Crohn's disease were rapidly obscured by the report of a high incidence of NRH during treatment. In a study of 111 patients treated with $6-\mathrm{TG}, 26 \%$ presented with biological abnormalities (thrombopenia and abnormal functional liver tests) which evoked NRH. Liver puncture biopsy confirmed this diagnosis in $76 \%$ of this patient group and in $33 \%$ of totally asymptomatic patients who had agreed to undergo biopsy. ${ }^{20}$ Following that publication, the use of 6-TG treatment in inflammatory bowel disease was suspended in most countries.

The onset of NRH in patients treated with 6-TG has been attributed to high levels of 6-TGN, the active principle of thiopurine therapy. Generally, 6-TGN levels are substantially higher with 6-TG treatment than with azathioprine/6-MP. ${ }^{20}$ Nevertheless, few data are available concerning hepatic tolerance of azathioprine/6-MP at currently recommended doses (2-3 mg/kg daily for azathioprine, $1-1.5 \mathrm{mg} / \mathrm{kg}$ daily for 6-MP). As azathioprine is used increasingly often as maintenance treatment in inflammatory bowel disease, and is given earlier in the course of disease and for longer periods, the risk of NRH must be more thoroughly elucidated. The objective of this multicentre study was to evaluate the prevalence of NRH and its characteristics, outcome, and risk factors in patients with inflammatory bowel disease treated with azathioprine/6-MP.

\section{METHODS}

All gastroenterologists from GETAID (35 centres in Belgium, France, and Switzerland) and from one centre in Austria were contacted and requested to notify NRH cases observed in their patients with inflammatory bowel disease in whom azathioprine/ 6-MP was initiated between May 1985 and August 2004. Data from each patient were collected using a standardised questionnaire.

\section{Questionnaire}

Clinical descriptive data, pathological changes in laboratory indices-including liver enzymes, thiopurine methyl transfer-

Abbreviations: $\mathrm{NRH}$, nodular regenerative hyperplasia; TIPS, transjugular intrahepatic portosystemic shunt; 6-MP, 6-mercaptopurine; 6-TG, 6thioguanine; 6-TGN, 6-thioguanine nucleotides 
ase (TPMT) genotyping, and 6-TGN measurement when available-along with the histology of liver biopsies, were surveyed. Clinical data included the following: demographics; disease phenotype according to the Montreal classification ${ }^{21}$; disease duration; previous intestinal resection including large small bowel resections (defined as $\geqslant 50 \mathrm{~cm}$ ) occurring before azathioprine was started; indication for, dosage, and duration of azathioprine treatment; concomitant drug treatment; clinical symptoms at the time of NRH diagnosis; and all available follow up information concerning complications of liver disease and specific treatment.

All patients were required to undergo a liver biopsy. As a definite diagnosis of NRH on the basis of a single liver biopsy may be difficult, patients were classified as having either confirmed or suspected NRH according to the following criteria:

- Confirmed NRH: histological study of liver biopsy showing hyperplastic parenchymatous nodules without extensive fibrosis on silver reticulin staining, with compressed and atrophic internodular parenchyma.

- Suspected NRH: non-cirrhotic portal hypertension of unknown origin despite a battery of clinical, biological, and morphological investigations, associated with histological study of the liver showing irregularity of hepatic plates without true nodulation and annular fibrosis. In these cases, biopsy was assigned the nomenclature "equivocal pathohistological findings possibly related to $\mathrm{NRH}^{\prime \prime}$ by the pathologist.

\section{Data analysis and statistics}

All records were reviewed for accuracy and completeness and validated by the responsible investigator (GVM).

The cumulative risk of NRH was estimated using KaplanMeier estimates from 1958 consecutive patients with inflammatory bowel disease treated with azathioprine and followed at one centre (Saint-Antoine Hospital, Paris). Previous information and follow up data from Crohn's disease patients in this cohort have been published earlier. ${ }^{22}$ The median duration of azathioprine treatment in this cohort was 28 months (interquartile range (IQR), 10 to 59). Eleven cases of NRH were identified at the centre.

Risk factors for NRH were sought using the univariate and multivariate proportional hazards model, after stratification on duration of immunomodulators by forward selection though likelihood ratio for the latter. Cases comprised patients with $\mathrm{NRH}$ reported in this study. Controls were selected from the Saint-Antoine hospital's inflammatory bowel disease database (patients with inflammatory bowel disease who were treated with azathioprine without abnormal liver functional tests, $\mathrm{n}=1947$ ) and matched one to four to each case by the date of starting azathioprine. The same data were recorded in cases and controls at the time azathioprine was started. Variables tested included sex, age, inflammatory bowel disease phenotype, disease duration, perianal fistula, disease location and behaviour (for location and behaviour, patients with ulcerative colitis were included in the L2 group and the B1 group, respectively), previous intestinal resections, and previous large small bowel resections when starting azathioprine. In addition to the model selected using forward selection, other models with a lower but close likelihood were looked for in order to take account of the relatively small number of cases.

Data are presented as median (range or IQR) or as percentages. The cumulative risk of NRH and NRH complications is given with a $95 \%$ confidence interval (CI). A probability (p) value $<0.05$ is considered significant.

\section{RESULTS}

\section{Case characteristics}

Thirty seven cases of NRH (31 with Crohn's disease and six with ulcerative colitis) were observed between 1994 and 2005 at 11 centres (table 1) and included 30 male patients (81\%) and seven female patients (19\%). The median duration of disease before the time of NRH diagnosis was 15 years (range 1 to 39). Among the patients with Crohn's disease, 10 were smokers, seven were non-smokers, and nine were ex-smokers. Among the patients with ulcerative colitis, three were ex-smokers. Twenty three of the patients were operated on (22 patients with Crohn's disease and one with ulcerative colitis). Among the Crohn's disease patients, one had a colectomy and 21 had small bowel resection, 19 of them before azathioprine treatment, with large resections $(\geqslant 50 \mathrm{~cm})$ in 16 cases. Eleven of the 1958 patients followed at Saint-Antoine Hospital developed NRH. At that centre, the cumulative risk of NRH was $0.5 \%$ at five years after starting azathioprine (95\% CI, 0.11 to 0.89$)$, and $1.25 \%$ at 10 years $(0.29$ to 2.21$)$.

\section{Azathioprine treatment}

The median time between the start of azathioprine treatment and the discovery of NRH was 48 months (range 6 to 187) (table 2). Azathioprine was stopped in all except one patient before or at the time of the suspected diagnosis of NRH. Before being placed on azathioprine treatment, patients had received 5-ASA $(n=37)$, corticosteroids $(n=37)$, cyclosporin $(n=1)$, methotrexate for six months $(n=1)$, or infliximab $(n=2)$. At the time of NRH diagnosis, patients were being given azathioprine combined with 5-ASA $(n=2)$, corticosteroids $(n=12)$, infliximab $(n=3)$, and enteric feeding $(n=1)$.

\begin{tabular}{|c|c|}
\hline Variable & \\
\hline \multicolumn{2}{|l|}{$\operatorname{Sex}(n(\%))$} \\
\hline Male & $30(81)$ \\
\hline Female & 7 (19) \\
\hline \multicolumn{2}{|l|}{ Diagnosis (n (\%)) } \\
\hline Crohn's disease & $31(84)$ \\
\hline Ulcerative colitis & $6(16)$ \\
\hline \multicolumn{2}{|l|}{ Age at disease onset (years) } \\
\hline \multirow{2}{*}{\multicolumn{2}{|c|}{ Crohn's disease location* (n (\%)) }} \\
\hline & \\
\hline lleal L1 & $18(58)$ \\
\hline Colonic L2 & $4(13)$ \\
\hline Ileocolonic L3 & $7(23)$ \\
\hline Upper gastrointestinal L4 & $2(6)$ \\
\hline \multicolumn{2}{|l|}{ Crohn's disease behaviour* (n (\%)) } \\
\hline Non-stricturing, non-penetrating & $12(39)$ \\
\hline Stricturing & $12(39)$ \\
\hline Penetrating & $7(22)$ \\
\hline \multicolumn{2}{|l|}{ Ulcerative colitis extent ${ }^{\star}(\mathrm{n}(\%))$} \\
\hline Proctitis & $0(0)$ \\
\hline Left sided colitis & $3(50)$ \\
\hline Extensive colitis & $3(50)$ \\
\hline \multicolumn{2}{|l|}{$\begin{array}{l}\text { Crohn's disease: small bowel resection } \\
\text { before AZA treatment (n (\%)) }\end{array}$} \\
\hline Never & $12(39)$ \\
\hline$<50 \mathrm{~cm}$ & $3(10)$ \\
\hline$\geqslant 50 \mathrm{~cm}$ & $16(51)$ \\
\hline \multicolumn{2}{|l|}{ Smoking habit (n (\%)) } \\
\hline Smoker & $10(27)$ \\
\hline Ex-smoker & $12(32)$ \\
\hline Non-smoker & $7(19)$ \\
\hline Unknown & $8(22)$ \\
\hline
\end{tabular}




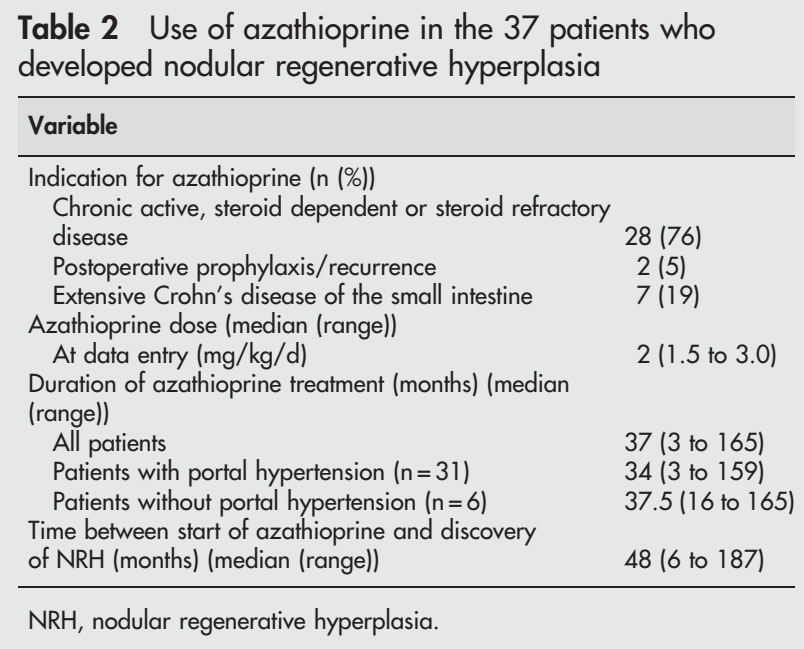

Seven patients underwent a search for inactivating mutations of the thiopurine S-methyltransferase gene (TPMT). All were homozygous for a functional allele of the gene. Seven patients underwent an assay of 6-TGN at the time of NRH diagnosis; the median value was $268 \mathrm{pmol} / 8 \times 10^{8}$ erythrocytes (range 68 to 625 ).

\section{NRH symptoms and outcome}

Median age at diagnosis of NRH was 36 years (range 18 to 70). For 12 patients, circumstances leading to the discovery of NRH included clinical symptoms or signs of portal hypertension (table 3). Six patients presented with variceal bleeding, four with ascites or oedema of the lower limbs, and two had splenomegaly. In four patients, portal hypertension was discovered by imaging which showed splenomegaly and portacaval collaterals. Twenty one patients presented with hepatic biological abnormalities $(n=5)$ and thrombocytopenia $(n=6)$, or both $(n=10)$. At diagnosis, four patients had isolated thrombocytopenia $\left(<150 \times 10^{9} / 1\right)$, five had isolated abnormal liver function tests (raised alanine aminotransferase (ALT), aspartate aminotransferase (AST), alkaline phosphatase, or bilirubin to $>1.2$ times the upper limit of normal range), 26 had both thrombocytopenia and abnormal liver tests, and two had no biological anomalies.

Twenty five patients presented with oesophageal varices at endoscopy. In all, 31 of the 37 patients had portal hypertension

Table 3 Characteristics of complications in the 37 patients who developed NRH

\begin{tabular}{|c|c|}
\hline Variable & Frequency \\
\hline \multicolumn{2}{|l|}{ Circumstances leading to the discovery of NRH (n (\%)) } \\
\hline Clinicals symptoms of portal hypertension & $12(32)$ \\
\hline Imaging of portal hypertension & $4(11)$ \\
\hline Biological abnormalities: hepato- and/or haemotoxicity* & $21(57)$ \\
\hline \multicolumn{2}{|l|}{ Liver biopsies } \\
\hline Confirmed cases of NRH (n (\%)) & $29(78)$ \\
\hline Suspected cases of NRH (n (\%)) & $8(22)$ \\
\hline \multicolumn{2}{|l|}{ Laboratory variables at diagnosis of $\mathrm{NRH}(\mathrm{n}(\%))$} \\
\hline No biological anomalies & $2(5)$ \\
\hline Liver abnormalities* & $5(14)$ \\
\hline Thrombocytopenia* & $4(11)$ \\
\hline Both* & $26(70)$ \\
\hline $\begin{array}{l}\text { Portal hypertension at diagnosis (endoscopy and/or liver } \\
\text { imaging) }(\mathrm{n}(\%) \text { ) }\end{array}$ & $31(84)$ \\
\hline \multicolumn{2}{|c|}{$\begin{array}{l}\text { *Raised alanine aminotransferase (ALT), aspartate aminotransferase (AST), } \\
\text { alkaline phosphatase, or bilirubin to }>1.2 \text { times the upper limit of normal } \\
\text { range; platelets }<150 \times 10^{9} / \text { l. } \\
\text { NRH, nodular regenerative hyperplasia. }\end{array}$} \\
\hline
\end{tabular}

at endoscopy or liver imaging, and only six had isolated biological abnormalities. The median duration of azathioprine treatment before the diagnosis of NRH was 34 months (range 3 to 159) for patients with portal hypertension, and 37.5 months (range 16 to 165) for patients without portal hypertension.

All patients underwent liver biopsy-16 by the percutaneous route, 18 by the transjugular route, and three during surgery, including one liver transplant. The median length of the biopsy fragment was $17 \mathrm{~mm}$ (range 7 to 26), and the median number of portal zones was 9 (range 2 to 20 ). In the 18 patients who had undergone transjugular assessment of hepatic pressure, the median value of the portacaval gradient $(n=10)$ was 12.5 mm Hg (range 2 to 29); in patients with symptoms or signs of portal hypertension, nine $(90 \%)$ had a high portacaval gradient (>4 mm Hg). There were 29 firmly confirmed cases of NRH on histological examination. Eight patients had suspected NRH with non-cirrhotic portal hypertension, with clinical complications in six, including rupture of oesophageal varices $(n=3)$ and ascites $(n=3)$. In 16 patients $(43 \%)$, hepatic vascular lesions were found associated with NRH lesions, including sinusoidal dilatations $(n=12)$, perisinusoidal fibrosis $(n=12)$, portal venopathies $(n=4)$, and peliosis $(n=1)$. Among other associated histological lesions, we noted six cases of hepatic steatosis and one of hepatoportal sclerosis.

The six patients without portal hypertension at diagnosis did not present with any complications during follow up. Two of these had normal liver function tests and normal platelet counts at 15 and 45 months after NRH diagnosis. Among the 31 patients presenting with portal hypertension at diagnosis, liver function tests normalised in two and the blood platelet count in six after a median follow up of 15 months (range 1 to 138). Twenty three had biological abnormalities at the most recent follow up. Complications of portal hypertension occurred in 14 patients, including nine with acute variceal bleeding and five with ascites. Cumulative survival without complications of portal hypertension from the time of diagnosis of NRH was $69 \%$ at three years (95\% CI, 27 to 100) (fig 1).

No deaths or hepatocellular carcinomas occurred. Fifteen patients underwent primary or secondary treatment for portal hypertension, including $\beta$ blockers and nitrous derivatives $(n=11)$, endoscopic therapy $(n=9)$, embolisation $(n=2)$, and transjugular intrahepatic portosystemic shunt (TIPS, $\mathrm{n}=2$ ). One patient underwent liver transplantation because of hepatic encephalopathy following TIPS insertion.

\section{Risk factors for NRH}

Characteristics of cases and controls are shown in table 4. Using univariate analysis, disease duration before treatment, male sex, age, ileal location L1, stricturing behaviour B2, and small and large bowel resection were risk factors for development of

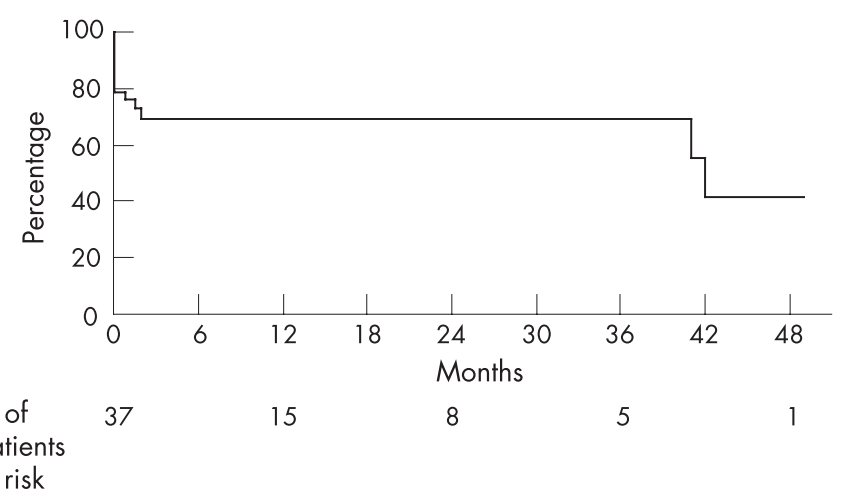

Figure 1 Cumulative survival without complications of portal hypertension from time of diagnosis of nodular regenerative hyperplasia in 37 patients. 
NRH. In multivariate analysis, only two variables remained significant: male sex (hazard ratio (HR) $=4.1$ (95\% CI, 1.8 to 9.5)) $(\mathrm{p}<0.001)$ and stricturing behaviour B2 $(\mathrm{HR}=3.0(95 \%$ CI, 1.4 to 6.4$)$ ) $(\mathrm{p}=0.006)$. Three other models provided a lower but close likelihood when adjusting the data. The risk factors included in these models were: male sex $(\mathrm{HR}=4.2,3.7$, or 3.9) combined with stricturing or penetrating behaviour $(\mathrm{HR}=3.4)$, and previous large small bowel resection $(\mathrm{HR}=2.5)$, or ileal location $(\mathrm{HR}=2.4)$.

\section{DISCUSSION}

We report here 37 cases of NRH which occurred during treatment with azathioprine in patients with inflammatory bowel disease. This is the largest series reported so far. The cumulative risk of NRH could be estimated at one centre as $0.50 \%$ at five years and $1.25 \%$ at 10 years. Despite the particularly high risk at this centre ( 11 of the 37 cases in our series), it remained notably lower than that observed with 6TG. ${ }^{20}$ However, it may have been underestimated, as only patients presenting with clinical symptoms or biological abnormalities were reported, and no screening procedures using laboratory tests or specific imaging techniques such as magnetic resonance imaging were systematically undertaken during the follow up. NRH is often asymptomatic. In two necropsy series, its prevalence varied between $0.6 \%$ and $2.6 \%$ of the cases studied ${ }^{43}$ in the absence of any previously known manifestation. In the study by Dubinsky et al, 33\% of patients with asymptomatic inflammatory bowel disease treated with 6TG and with normal biological results were shown to have NRH at liver biopsy. ${ }^{20}$

The diagnosis of NRH can be challenging. It is based on percutaneous or transjugular liver biopsy, which may not be accurate when the sample is small or fragmented. ${ }^{323-25}$ Interobserver disagreement between pathologists has been reported in the diagnosis of NRH. In our study, centralised re-examination of the biopsy specimen by the same pathologist was not undertaken, but strict histological criteria were used, including reticulin staining as recently recommended.

Our series was characterised by the severity of the observed cases. Thirty one patients presented with portal hypertension on diagnosis, with an actuarial complication rate of $31 \%$ at three years. Clinical progression was severe even after stopping azathioprine, and included several cases of ascites and rupture of oesophageal varices which, for one patient, necessitated liver transplantation. Such severity of portal hypertension associated with NRH has already been reported in patients treated with azathioprine following renal transplantation. ${ }^{1425}{ }^{26}$ In the five other reported cases of NRH during the course of azathioprine treatment for inflammatory bowel disease, the following were noted: rupture of the oesophageal varices on diagnosis, necessitating the insertion of a TIPS in one case ${ }^{16}$; rupture of the oesophageal varices during follow up, necessitating a portacaval shunt by the surgical pathway'; and icterus with hepatocellular carcinoma. ${ }^{17}$ There were no hepatocellular carcinomas in our study. Liver regeneration might enhance hepatic carcinogenesis during the course of $\mathrm{NRH}^{17} 2728$ and thus regular monitoring by liver echography and assay of $\alpha$ fetoprotein is recommended. It is noteworthy that in the six patients for whom NRH was diagnosed on the basis of biological anomalies in the absence of any clinical symptoms, no signs of portal hypertension were found during the course of follow up. These patients did not differ from the others in terms of the dose of azathioprine or length of treatment. Those six observations highlight the advantage of early diagnosis, which would allow azathioprine treatment to be halted before the onset of complications. Patients who are put on azathioprine should be informed of the risk of NRH and of the need to monitor liver function and platelet counts. When biological anomalies appear, cessation of azathioprine treatment is required.

In this series, we did not observe NRH in any patient on 6MP. Several cases of NRH have been reported in patients with inflammatory bowel disease treated with $6-\mathrm{TG}^{20}$ eight cases with azathioprine, ${ }^{916-19}$ but no case with 6-MP. Cases of NRH have also been reported in renal transplant patients and children with acute lymphoblastic leukaemia treated with azathioprine or 6-TG. ${ }^{6-12} 29$ This disproportion between azathioprine and 6-MP probably reflects the much wider use of azathioprine than 6-MP in inflammatory bowel disease. Indeed, both drugs (like 6-TG) share the same putative toxic metabolites, 6-TGN. ${ }^{30}$

Our case-control analysis had some limitations. Controls were recruited only from one centre, while cases were from 11 participating centres. However, the nature of the practice (tertiary care referral centres) was similar in all. In addition, the time of follow up differed between cases and controls. Nevertheless, it is unlikely that loss of follow up in controls was secondary to $\mathrm{NRH}$, and stratification was carried out on the duration of azathioprine treatment. Using multivariate analysis, two risk factors for NRH in patients receiving azathioprine were identified: male sex and stricturing behaviour. Four models, including gender associated with disease behaviour or location or resection, provided very similar data adjustment according to likelihood estimates. This is not surprising, as these three variables were highly correlated $(\mathrm{p}<0.0001$ for all pairs). Therefore, it should be emphasised that selection of the factor

Table 4 Clinical characteristics of patients with nodular regenerative hyperplasia and matched controls at initiation of azathioprine treatment

\begin{tabular}{|c|c|c|c|}
\hline Variable & $\begin{array}{l}\text { Patients with NRH } \\
(\mathrm{n}=37)\end{array}$ & $\begin{array}{l}\text { Control patients } \\
(n=148)\end{array}$ & p Value \\
\hline Age (years) (median (IQR)) & $36(24$ to 43$)$ & $30(23$ to 41$)$ & NS \\
\hline Disease duration (months) (median (IQR)) & 81 (34 to 172$)$ & 50 (14 to 87$)$ & 0.001 \\
\hline IBD phenotype (Crohn's disease/ulcerative colitis) & $31 / 6$ & $127 / 21$ & NS \\
\hline Sex (male/female) & $30 / 7$ & $58 / 90$ & $<0.001$ \\
\hline Duration of immunomodulator treatment (months) (median (range)) & $37(3$ to 165$)$ & $33(1.5$ to 160$)$ & 0.001 \\
\hline \multicolumn{4}{|l|}{ Disease location } \\
\hline $\mathrm{L} 1 / \mathrm{L} 2 * / \mathrm{L} 3 / \mathrm{L} 4$ & $18 / 10 / 7 / 2$ & $29 / 62 / 46 / 11$ & 0.01 \\
\hline Perianal fistula (Crohn's disease only) (yes/no) & $6 / 25$ & $33 / 94$ & NS \\
\hline \multicolumn{4}{|l|}{ Disease behaviour (Crohn's disease only) } \\
\hline $\mathrm{B} 1 / \mathrm{B} 2 / \mathrm{B} 3$ & $12 / 12 / 7$ & $90 / 15 / 22$ & 0.002 \\
\hline Previous intestinal resection (yes/no) & $19 / 18$ & $33 / 115$ & $<0.001$ \\
\hline Previous large small bowel resection (yes/no) & $16 / 21$ & $19 / 129$ & $<0.001$ \\
\hline
\end{tabular}

*Patients with ulcerative colitis were included in the $\mathrm{L} 2$ group and the $\mathrm{B} 1$ group.

$I B D$, inflammatory bowel disease; IQR, interquartile range; $N R H$, nodular regenerative hyperplasia. 
associated with gender in the best model (with maximum likelihood) may be highly dependent on case sampling because of the limited number of cases. The male sex specificity of this complication has already been pointed out. All children who developed NRH when receiving 6-TG during maintenance therapy for acute lymphoblastic leukaemia were boys. ${ }^{29}$

The link between stricturing behaviour and NRH remains unknown. The pathophysiology of NRH is poorly understood. It could result from heterogeneous perfusion of the hepatic parenchyma, causing hepatocytic atrophy in hypoperfused areas and compensatory hyperplasia in those which remain normally vascularised. ${ }^{31} \mathrm{NRH}$ associated with azathioprine might be secondary not to portal venule lesions but rather to sinusoidal lesions, through depletion of cells of glutathione ${ }^{32}$ and eventually to the formation of tiny extrahepatic veins which are provoked by this drug (sinusoidal dilatation, peliosis, perisinusoidal fibrosis, and veno-occlusive disease). ${ }^{11} \mathrm{NRH}$ has been associated with thrombotic risk factors such as hyperhomocysteinaemia. ${ }^{33}$ In this context, it can be speculated that a deficit in vitamin B-6, folates, and vitamin B-12 secondary to ileal stenosis (strongly associated with an increased risk of intestinal resection) could favour the onset of hepatic vascular lesions through the occurrence of hyperhomocysteinaemia. It has been proposed that the development of NRH may be dependent on high levels of 6-TGN. ${ }^{34}$ In our patients, the dose of azathioprine used was a standard one (an average of $2 \mathrm{mg} /$ $\mathrm{kg}$ ), and in the seven patients for whom this assay was available, concentrations of 6-TGN were within the usual therapeutic range in all but one case. Nor does NRH seem to be linked to long term drug exposure: over half of the cases arose within three years of treatment. In the six patients who presented without portal hypertension, the dose and duration of treatment were not lower or shorter than in the other cases.

Treatment with azathioprine might be associated with the onset of NRH in the course of inflammatory bowel disease. The frequency of this complication is rare, but it is probably underestimated owing to its non-specific mode of discovery (anomalies revealed at liver biological monitoring, or thrombopenia), and the frequency of totally asymptomatic forms. Because it is poorly understood, NRH is most often diagnosed following severe portal hypertension, which could have been avoided by early diagnosis. Recent studies using magnetic resonance imaging are promising, but need to be confirmed in the diagnosis of NRH. ${ }^{35}$ Our series illustrates the importance for clinicians of regular monitoring of functional liver tests and platelet counts in patients under azathioprine treatment for Crohn's disease, especially in the presence of risk factors such as male sex, stricturing behaviour, and small bowel resection.

\section{ACKNOWLEDGEMENTS}

We wish to thank all participants, with special thanks to Patricia Détré from the Groupe d'Etudes Thérapeutiques des Affections Inflammatoires du Tube Digestif (GETAID), Dr Paupard, and Dr Balavoine.

\footnotetext{
Authors' affiliations

Gwenola Vernier-Massouille, Jean-Frédéric Colombel, Department of Hepato-Gastroenterology, Hôpital Huriez, Lille, France Jacques Cosnes, Philippe Seksik, Department of HepatoGastroenterology, Hôpital Saint Antoine, Paris, France

Marc Lemann, Department of Hepato-Gastroenterology, Hôpital Saint Louis, Paris, France

Philippe Marteau, Department of Hepato-Gastroenterology, Hôpital Lariboisière, Paris, France

Walter Reinisch, Department of Hepato-Gastroenterology, University of Vienna, Austria

David Laharie, Department of Hepato-Gastroenterology, Hôpital HautLeveque, Bordeaux, France
}

Guillaume Cadiot, Department of Hepato-Gastroenterology, Hôpital Robert Debré, Reims, France

Yoram Bouhnik, Department of Hepato-Gastroenterology, Hôpital Beaujon, Paris, France

Martine De Vos, Department of Hepato-Gastroenterology, University of Gand, Belgium

Arnaud Boureille, Department of Hepato-Gastroenterology, Hôtel Dieu, Nantes, France

Bernard Duclos, Department of Hepato-Gastroenterology, Hôpital Universitaire de Hautepierre, Strasbourg, France

Jean-Yves Mary, Department of Hepato-Gastroenterology, Inserm U717, Université Paris 7, Paris

Conflict of interest: None declared.

\section{REFERENCES}

1 Steiner PE. Nodular regenerative hyperplasia of the liver. Am J Pathol 1959;35:943-53.

2 Stromeyer FW, Ishak KG. Nodular transformation (nodular "regenerative" hyperplasia) of the liver. A clinicopathologic study of 30 cases. Hum Pathol $1981 ; 12: 60-71$.

3 Arvanitaki M, Adler M. Nodular regenerative hyperplasia of the liver. A review of 14 cases. Hepatogastroenterology $2001 ; 48: 1425-9$.

4 Wanless IR, Godwin TA, Allen F, et al. Nodular regenerative hyperplasia of the liver in hematologic disorders: a possible response to obliterative portal venopathy. A morphometric study of nine cases with an hypothesis on the pathogenesis. Medicine 1980;59:367-79.

5 Naber AH, Van Haelst U, Yap SH. Nodular regenerative hyperplasia of the liver: an important cause of portal hypertension in non-cirrhotic patients. J Hepatol $1991 ; 12: 94-9$.

6 Buffet C, Cantarovitch M, Pelletier G, et al. Three cases of nodular regenerative hyperplasia of the liver following renal transplantation. Nephrol Dial Transplant 1988;3:327-30.

7 Haboubi NY, Ali HH, Whitwell HL, et al. Role of endothelial cell injury in the spectrum of azathioprine-induced liver disease after renal transplant: light microscopy and ultrastructural observations. Am J Gastroenterol 1988;83:256-61.

8 Jones MC, Best PV, Catto GR. Is nodular regenerative hyperplasia of the liver associated with azathioprine therapy after renal transplantation? Nephrol Dial Transplant 1988;3:331-3.

9 Duvoux C, Kracht M, Lang P, et al. [Nodular regenerative hyperplasia of the liver associated with azathioprine therapy. ] Gastroenterol Clin Biol, $1991 ; 15: 968-73$

10 Bredfeldt JE, Havey AL. Nodular regenerative hyperplasia of the liver following renal transplantation. Dig Dis Sci 1981;26:271-4.

11 Katzka DA, Saul SH, Jorkasky D, et al. Azathioprine and hepatic venocclusive disease in renal transplant patients. Gastroenterology 1986;90:446-54.

12 Morales JM, Prieto C, Colina F, et al. Nodular regenerative hyperplasia of the liver in renal transplantation. Transplant Proc 1987; 19:3694-6.

13 Colina F, Alberti N, Solis JA, et al. Diffuse nodular regenerative hyperplasia of the liver (DNRH). A clinicopathologic study of 24 cases. Liver 1989;9:253-65.

14 Radomski JS, Chojnacki KA, Moritz MJ, et al. Results of liver transplantation for nodular regenerative hyperplasia. Am Surg 2000;66:1067-70.

15 Gisbert JP, Gonzalez-Lama Y, Mate J. Thiopurine-induced liver injury in patients with inflammatory bowel disease: a systematic review. Am J Gastroenterol 2007;27:27.

16 Arnott ID, Ghosh S. Portal hypertension in the presence of minimal liver damage in Crohn's disease on long-term azathioprine: possible endothelial cell injury. Eur J Gastroenterol Hepatol 2000;12:569-73.

17 Russmann S, Zimmermann A, Krahenbuhl S, et al. Veno-occlusive disease, nodular regenerative hyperplasia and hepatocellular carcinoma after azathioprine treatment in a patient with ulcerative colitis. Eur J Gastroenterol Hepatol 2001;13:287-90.

18 Daniel F, Cadranel JF, Seksik P, et al. Azathioprine induced nodular regenerative hyperplasia in IBD patients. Gastroenterol Clin Biol 2005;29:600-3.

19 Seiderer J, Zech CJ, Diebold J, et al. Nodular regenerative hyperplasia: a reversible entity associated with azathioprine therapy. Eur J Gastroenterol Hepatol 2006;18:553-5.

20 Dubinsky MC, Vasiliauskas EA, Singh $\mathrm{H}$, et al. 6-thioguanine can cause serious liver injury in inflammatory bowel disease patients. Gastroenterology 2003;125:298-303.

21 Satsangi J, Silverberg MS, Vermeire S, et al. The Montreal classification of inflammatory bowel disease: controversies, consensus, and implications. Gut 2006;55:749-53.

22 Cosnes J, Nion-Larmurier I, Beaugerie L, et al. Impact of the increasing use of immunosuppressants in Crohn's disease on the need for intestinal surgery. Gut 2005;54:237-41.

23 Wanless IR, Peterson P, Das A, et al. Hepatic vascular disease and portal hypertension in polycythemia vera and agnogenic myeloid metaplasia: a clinicopathological study of 145 patients examined at autopsy. Hepatology 1990;12:1166-74.

24 Nakanuma $Y$, Hoso M, Sasaki M, et al. Histopathology of the liver in noncirrhotic portal hypertension of unknown aetiology. Histopathology 1996;28:195-204. 
25 Blanc JF, Bernard PH, Le Bail B, et al. [Vascular pathology of the portal vein distal branches: a rare cause of liver transplantation and a protean clinical presentation. ] Gastroenterol Clin Biol, 2000;24:667-70.

26 Elariny HA, Mizrahi SS, Hayes DH, et al. Nodular regenerative hyperplasia: a controversial indication for orthotopic liver transplantation. Transpl Int 1994;7:309-13.

27 Nzeako UC, Goodman ZD, Ishak KG. Hepatocellular carcinoma and nodular regenerative hyperplasia: possible pathogenetic relationship. Am J Gastroenterol 1996;91:879-84.

28 Snover DC. Thoughts on the development of hepatocellular carcinoma in cirrhotic and noncirrhotic livers. Am J Clin Pathol 1996;105:3-5.

29 Stoneham S, Lennard L, Coen $\mathrm{P}$, et al. Veno-occlusive disease in patients receiving thiopurines during maintenance therapy for childhood acute lymphoblastic leukaemia. Br J Haematol 2003;123:100-2.

30 Dubinski MC. Azathioprine, 6-mercaptopurine in inflammatory bowel disease: pharmacology, efficacy, and safety. Clin Gastroenterol Hepatol 2004;2:731-43.
31 Wanless IR. Micronodular transformation (nodular regenerative hyperplasia) of the liver: a report of 64 cases among 2,500 autopsies and a new classification of benign hepatocellular nodules. Hepatology 1990;11:787-97.

32 DeLeve LD, Wang X, Kuhlenkamp JF, et al. Toxicity of azathioprine and monocrotaline in murine sinusoidal endothelial cells and hepatocytes: the role of glutathione and relevance to hepatic venoocclusive disease. Hepatology 1996;23:589-99.

33 Buchel O, Roskams T, Van Damme B, et al. Nodular regenerative hyperplasia, portal vein thrombosis, and avascular hip necrosis due to hyperhomocysteinaemia. Gut 2005;54:1021-3.

34 De Boer NK, Mulder CJ, Van Bodegraven AA. Nodular regenerative hyperplasia and thiopurines: the case for level-dependent toxicity. Liver Transpl 2005;11:1300-1.

35 Seiderer J, Zech CJ, Reinisch W, et al. A multicenter assessment of liver toxicity by MRI and biopsy in IBD patients on 6-thioguanine. J Hepatol 2005:43:303-9.

\section{EDITOR'S QUIZ: GI SNAPSHOT}

\section{Jaundice in a chronic hepatitis B carrier}

\section{Clinical presentation}

A 50-year-old man presented with a 4-week history of jaundice that developed progressively. He had chronic hepatitis B. During the last 3 months, he had complained of repetitive bouts of right upper abdominal pain with fever. Clinical examination was normal except for jaundice. Biological tests revealed a normal complete blood count and abnormal liver tests with cholestatic and cytolytic features ( $\gamma$-glutamyl transpeptidase $=850 \mathrm{IU} / \mathrm{l}$ $($ normal $<43 \mathrm{IU} / \mathrm{l})$, alkaline phosphatase $=299 \mathrm{IU} / \mathrm{l}$ (normal $<126)$, total bilirubin $=134 \mu \mathrm{m} / \mathrm{l} \quad($ normal $<22)$, aspartate

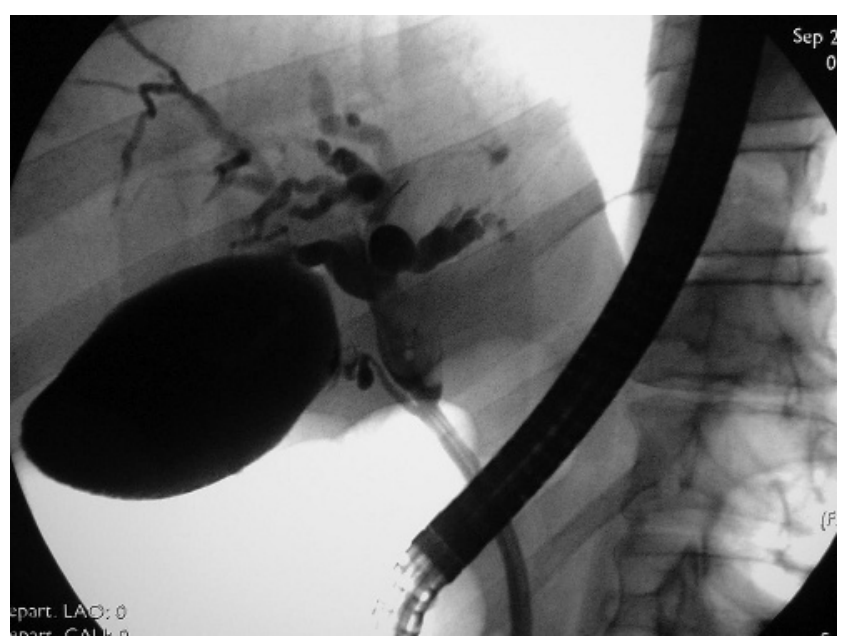

Figure 1 Cholangiogram through the ERCP catheter inserted into the common bile duct up to the junction with the cystic duct. There is aneurysmal dilation of the main hepatic duct containing a filling defect with dilated right intrahepatic ducts. The left intrahepatic duct is not visualised. transaminase $=101 \mathrm{IU} / 1$ (normal $<59)$, alanine transaminase $=$ 116 IU/l (normal $<72$ ). The $\alpha$-fetoprotein level was normal and the CA 19-9 level was elevated at $139 \mathrm{IU} / \mathrm{ml}$ (normal<37 IU/ml). HBsAg and IgG anti-HBc were positive.

On abdominal ultrasonography, the intrahepatic bile ducts were dilated. No parenchymal tumour was detected and the gallbladder was unremarkable. Magnetic resonance cholangiopancreatography showed a homogeneous liver parenchyma and a filling defect of the hepatic duct with upward dilatation. Endoscopic retrograde cholangiography was carried out and is shown in fig 1 .

\section{Question}

What is the diagnosis and management?

See page 1425 for answer

R Noun de France Hospital, Beirut, Lebanon

S Zeidan

Department of Digestive Surgery, Hotel Dieu de France Hospital, Beirut,

Lebanon

C Ghorra

Department of Pathology, Hotel Dieu de France Hospital, Beirut, Lebanon

S Slaba

Saint Joseph University of Beirut, Department of Radiology, Hotel Dieu de

France Hospital, Beirut, Lebanon

L Menassa-Moussa

Department of Radiology, Hotel Dieu de France Hospital, Beirut, Lebanon

R Sayegh

Saint Joseph University of Beirut, Department of Gastroenterology, Hotel Dieu de France Hospital, Beirut, Lebanon

Correspondence to: Dr S Zeidan, Department of Digestive Surgery, Hotel Dieu de France Hospital, Alfred Naccache Street, Achrafieh, PO Box 166830, Beirut, Lebanon; smart_zeidan@yahoo.com doi: $10.1136 /$ gut.2006.103887 